International Journal of Mathematical Analysis

Vol. 9, 2015, no. 2, 81 - 90

HIKARI Ltd, www.m-hikari.com

http://dx.doi.org/10.12988/ijma.2015.46186

\title{
Common Fixed Point Theorems of $R$-weakly Commuting Mappings in Fuzzy Metric Spaces
}

\author{
Shin Min Kang \\ Department of Mathematics and RINS \\ Gyeongsang National University \\ Jinju 660-701, Korea \\ Vishal Gupta and Balbir Singh \\ Department of Mathematics \\ Maharishi Markandeshwar University \\ Mullana, Ambala, Haryana, India \\ Sanjay Kumar \\ Department of Mathematics \\ Deenbandhu Chhotu Ram University of Science and Technology \\ Murthal, Sonipat, Haryana, India
}

Copyright (c) 2014 Shin Min Kang et al. This is an open access article distributed under the Creative Commons Attribution License, which permits unrestricted use, distribution, and reproduction in any medium, provided the original work is properly cited.

\begin{abstract}
In this paper, we prove a common fixed point theorems using $R$-weak commutativity in fuzzy metric spaces.
\end{abstract}

Mathematics Subject Classification: 47H10, 54H25

Keywords: Compatible mapping, reciprocal continuity, $R$-weakly commuting mapping, $t$-norm of Hadžić-type 


\section{Introduction}

Fuzzy set theory has many applications in applied sciences such as neural network theory, stability theory, mathematical programming, modelling theory, engineering sciences, medical sciences (medical genetics, nervous system), image processing, control theory, communication etc. Various authors such as Erceg [1], George and Veermani [2], Kaleva and Seikkala [6], Kramosil and Michálek [7] introduced the concept of fuzzy metric spaces in different ways. Here we are considering the fuzzy metric space in the sense of Kramosil and Michálek [7].

Definition 1.1. A binary operation $\Delta$ on $[0,1]$ is a $t$-norm if it satisfies the following conditions:

(i) $\Delta$ isassociative and commutative,

(ii) $\Delta(a, 1)=a$ for every $a \in[0,1]$,

(iii) $\Delta(a b) \leq \Delta(c, d)$, whenever $a \leq c$ and $b \leq d$.

Basics examples of $t$-norm are the Lukasiewicz $t$-norm $\Delta_{L}, \Delta_{L}(a, b)=$ $\max \{a+b-1,0\}$, the product $t$-norm $\Delta_{P}, \Delta_{P}(a, b)=a b$ and the minimum $t$-norm $\Delta_{M}, \Delta_{M}(a, b)=\min \{a, b\}$.

Definition 1.2. ([4]) Let $\Delta$ be a $t$-norm and $\Delta_{n}:[0,1] \rightarrow[0,1](n \in \mathbb{N})$ be defined by

$$
\Delta_{1}(x)=\Delta(x, x), \quad \Delta_{n+1}(x)=\Delta\left(\Delta_{n}(x), x\right) \quad(n \in \mathbb{N}, x \in[0,1]) .
$$

Then we say that the $t$-norm $\Delta$ is of Hadžić-type if the family $\left\{\Delta_{n}(x) ; n \in\right.$ $\mathbb{N}\}$ is equicontinuous at $x=1$. The family $\left\{\Delta_{n}(x) ; n \in \mathbb{N}\right\}$ is said to be equicontinuous at $x=1$ if for every $\lambda \in(0,1)$, there exists $\delta(\lambda) \in(0,1)$ such that

$$
x>1-\delta(\lambda) \text { implies } \Delta_{n}(x)>1-\lambda \quad(n \in \mathbb{N}) .
$$

A trivial example of $t$-norm of Hadžić-type is $\Delta=\Delta_{M}$.

Remark 1.3. ([4]) (1) If there exists a strictly increasing sequence $\left\{b_{n}\right\}_{n \in \mathbb{N}}$ in $[0,1]$ such that $\lim _{n \rightarrow \infty} b_{n}=1$ and $\Delta\left(b_{n}, b_{n}\right)=b_{n}$ for all $n \in \mathbb{N}$, then $\Delta$ is of Hadžić-type.

(2) If $\Delta$ is continuous and of Hadžić-type, then there exists a sequence $\left\{b_{n}\right\}_{n \in \mathbb{N}}$ as in (1).

Definition 1.4. ([4]) If $\Delta$ is a $t$-norm and $\left(x_{1}, x_{2}, x_{3}, \ldots, x_{n}\right) \in[0,1]^{n}(n \in$ $\mathbb{N})$, then $\Delta_{i=1}^{n} x_{i}$ is defined recurrely by 1 if $n=1$ and $\Delta_{i=1}^{n} x_{i}=\Delta\left(\Delta_{i=1}^{n-1} x_{i}, x_{n}\right)$ for all $n \geq 2$. If $\left\{x_{n}\right\}_{n \in \mathbb{N}}$ is a sequence in [0,1], then $\Delta_{i=1}^{\infty} x_{i}$ is defined as $\lim _{n \rightarrow \infty} \Delta_{i=1}^{n} x_{i}$ (this limit always exists) and $\Delta_{i=1}^{\infty} x_{i}$ as $\Delta_{i=1}^{\infty} x_{n+i}$. 
Definition 1.5. The 3-triple $(X, M, \Delta)$ is called a fuzzy metric space in the sence of Kramosil and Michálek if $X$ is an arbitrary set, $\Delta$ is a continuous $t$-norm and $M$ is a fuzzy set on $X^{2} \times[0, \infty)$ satisfying the following conditions: For all $x, y, z \in X$ and $s, t>0$

(FM-1) $M(x, y, 0)=0$,

(FM-2) $M(x, y, t)=1$ for all $t>0$ if and only if $x=y$,

(FM-3) $M(x, y, t)=M(y, x, t)$,

(FM-4) $M(x, z, t+s) \geq \Delta(M(x, y, t), M(y, z, s))$,

$(\mathrm{FM}-5) M(x, y, \cdot):[0, \infty) \rightarrow[0,1]$ is left continuous.

Note that $M(x, y, t)$ can be thought of as the degree of nearness between $x$ and $y$ with respect to $t$.

Remark 1.6. ([3]) In a fuzzy metric space $(X, M, \Delta), M(x, y, t)$ is nondecreasing with respect to $t$ for all $x, y \in X$.

Definition 1.7. A sequence $\left\{x_{n}\right\}$ in a fuzzy metric space $(X, M, \Delta)$ is said to be

(1) convergent to the limit $x$ if $\lim _{n \rightarrow \infty} M\left(x_{n}, x, t\right)=1$ for all $t>0$.

(2) Cauchy sequence in $X$ if for every $\lambda \in(0,1)$ and $t>0$, there exists a positive integer $N$ such that $M\left(x_{n}, x_{m}, t\right)>1-\lambda$ whenever $m, n \geq N$.

(3) complete if every Cauchy sequence in $X$ is convergent in $X$.

Fixed point theory in fuzzy metric spaces has been developing since the paper of Grabiec [3]. Subramanian [11] gave a generalization of Jungck's theorem ([5]) for commuting mapping in the setting of fuzzy metric spaces.

In 1994, Mishra et al. [8] generalised the notion of weakly commuting to compatible mappings in fuzzy metric spaces akin to the concept of compatible mapping in metric spaces (see [5]).

Definition 1.8. Let $f$ and $g$ be self-mappings on a fuzzy metric space $(X, M, \Delta)$. Then a pair $(f, g)$ is said to be compatible if $\lim _{n \rightarrow \infty} M\left(f g x_{n}, g f x_{n}, t\right)$ $=1$, whenever $\left\{x_{n}\right\}$ is a sequence in $X$ such that $\lim _{n \rightarrow \infty} f x_{n}=\lim _{n \rightarrow \infty} g x_{n}=$ $u$ for some $u \in X$ and for all $t>0$.

In 1994, Pant [9] introduced the concept of $R$-weakly commuting maps in metric spaces. Later on, Vasuki [12] introduced the notion of $R$-weakly commuting mapping in fuzzy metric spaces and proved some common fixed point theorems for these mappings.

Definition 1.9. Let $f$ and $g$ be self-mappings on a fuzzy metric space $(X, M, \Delta)$. Then a pair $(f, g)$ is said to be

(1) weakly commuting if $M(f g x, g f x, t) \geq M(f x, g x, t)$ for all $x \in X$ and $t>0$.

(2) $R$-weakly commuting if there exists $R>0$ such that $M(f g x, g f x, t) \geq$ $M(f x, g x, t / R)$ for all $x \in X$ and $t>0$. 
In 1999, Pant [10] introduced a new continuity condition, known as reciprocal continuity as follows.

Definition 1.10. Let $f$ and $g$ be self-mappings on a fuzzy metric space $(X, M, \Delta)$. Then $f$ and $g$ are called reciprocally continuous if $\lim _{n \rightarrow \infty} f g x_{n}=$ $f z$ and $\lim _{n \rightarrow \infty} g f x_{n}=g z$, whenever $\left\{x_{n}\right\}$ is a sequence such that $\lim _{n \rightarrow \infty} f x_{n}$ $=\lim _{n \rightarrow \infty} g x_{n}=z$ for some $z \in X$.

If $f$ and $g$ are both continuous, then they are obviously reciprocally continuous, but the converse is need not be true.

Lemma 1.11. ([8]) Let $(X, M, \Delta)$ be a fuzzy metric space. If there exists $q \in(0,1)$ such that $M(x, y, q t) \geq M(x, y, t)$ for all $x, y \in X$ and $t>0$, then $x=y$.

\section{Main Results}

Lemma 2.1. Let $(X, M, \Delta)$ be a fuzzy metric space with continuous t-norm of Hadžć-type. Let $A, B, S$ and $T$ be self-mappings on $X$ satisfying the following conditions:

$$
A(X) \subset T(X), \quad B(X) \subset S(X)
$$

(C2) there exists $q \in(0,1)$ such that

$$
\begin{gathered}
M(A x, B y, q t) \geq \min \{M(T y, B y, t), M(S x, A x, t), M(S x, B y,(2-\alpha) t), \\
M(T y, A x, \alpha t), M(T y, S x, t)\}
\end{gathered}
$$

for every $x, y \in X$ and $t>0$, where $\alpha \in(0,2)$. Then the continuity of one of the mappings in compatible pairs $(A, S)$ or $(B, T)$ on $(X, M, \Delta)$ implies their reciprocal continuity.

Proof. First assume that $A$ and $S$ are compatible and $S$ is continuous. We show that $A$ and $S$ are reciprocally continuous. Let $\left\{x_{n}\right\}$ be a sequence such that $\lim _{n \rightarrow \infty} A x_{n}=\lim _{n \rightarrow \infty} S x_{n}=z$ for some $z \in X$. Since $S$ is continuous, we have $\lim _{n \rightarrow \infty} S A x_{n}=S z$ and $\lim _{n \rightarrow \infty} S S x_{n}=S z$ and since $(A, S)$ is compatible, we have for all $t>0, \lim _{n \rightarrow \infty} M\left(A S x_{n}, S A x_{n}, t\right)=1$ and hence $\lim _{n \rightarrow \infty} A S x_{n}=S z$.

By $(C 1)$, for each $n$, there exists $\left\{y_{n}\right\}$ in $X$ such that $A S x_{n}=T y_{n}$. Thus, we have

$$
\lim _{n \rightarrow \infty} S S x_{n}=\lim _{n \rightarrow \infty} S A x_{n}=\lim _{n \rightarrow \infty} A S x_{n}=S z
$$

and $\lim _{n \rightarrow \infty} T y_{n}=S z$. 
Now we claim that $\lim _{n \rightarrow \infty} B y_{n}=S z$. Take $\alpha=1$ in $(C 2)$, we have

$$
\begin{aligned}
M\left(A S x_{n}, B y, q t\right) \\
\geq \min \left\{M\left(T y_{n}, B y_{n}, t\right), M\left(S S x_{n}, A S x_{n}, t\right), M\left(S S x_{n}, B y_{n}, t\right),\right. \\
\left.\quad M\left(T y_{n}, A S x_{n}, t\right), M\left(T y_{n}, S S x_{n}, t\right)\right\}
\end{aligned}
$$

Taking limit as $n \rightarrow \infty$ and using Lemma 1.11, we have $\lim _{n \rightarrow \infty} B y_{n}=S z$.

Next we claim that $A z=S z$. Again take $\alpha=1$ in $(C 2)$, we have

$$
\begin{gathered}
M\left(A z, B y_{n}, q t\right) \geq \min \left\{M\left(T y_{n}, B y_{n}, t\right), M(S z, A z, t), M\left(S z, B y_{n}, t\right),\right. \\
\left.M\left(T y_{n}, A z, t\right), M\left(T y_{n}, S z, t\right)\right\}
\end{gathered}
$$

Taking limit as $n \rightarrow \infty$, we have

$$
\begin{aligned}
M(A z, S z, q t) \geq \min \{ & M(S z, S z, t), M(S z, A z, t), M(S z, S z, t), \\
& M(S z, A z, t), M(S z, S z, t)\} \\
= & \min \{1, M(S z, A z, t), 1, M(S z, A z, t), 1\} \\
\geq & M(S z \cdot A z, t) .
\end{aligned}
$$

By Lemma 1.11, we have $A z=S z$. Therefore, $\lim _{n \rightarrow \infty} S A x_{n}=S z$ and $\lim _{n \rightarrow \infty} A S x_{n}=S z=A z$. Hence, $A$ and $S$ are reciprocally continuous on $X$.

If the pair $(B, T)$ is compatible and $\mathrm{T}$ is continuous. The proof is similar. This completes the proof.

Theorem 2.2. Let $(X, M, \Delta)$ be a fuzzy metric space with continuous minimum t-norm of Hadžić-type. Let $A, B, S$ and $T$ be self-mappings on $X$ satisfying $(C 1),(C 2)$ and the following conditions:

(C3) If one of the mappings in compatible pair $(A, S)$ or $(B, T)$ is continuous, $(C 4)$ the paira $(A, S)$ and $(B, T)$ are $R$-weakly commuting mappings.

Assume that there exist $x_{0}, x_{1} \in X$ such that for $y_{1}=A x_{0}=T x_{1}, y_{2}=$ $B x_{1}=S x_{2}$ and $\mu \in(q, 1)$

$$
\lim _{n \rightarrow \infty} \Delta_{i=n}^{\infty} M\left(y_{1}, y_{2}, 1 / \mu^{i}\right)=1 .
$$

Then $A, B, S$ and $T$ have a unique common fixed point in $X$.

Proof. Since $B(X) \subset S(X)$, there exist $x_{1}, x_{2} \in X$ such that $B x_{1}=S x_{2}$. Inductively, we can construct two sequences $\left\{x_{n}\right\}$ and $\left\{y_{n}\right\}$ of $X$ such that

$$
y_{2 n-1}=T x_{2 n-1}=A x_{2 n-2}, \quad y_{2 n}=S x_{2 n}=B x_{2 n-1}
$$

for $n=1,2, \ldots$ 
Take $\alpha=1-\beta$ in $(C 2)$, since $\Delta(a, b)=\min \{a, b\}$, we have

$$
\begin{aligned}
& M\left(y_{2 n+1}, y_{2 n+2}, q t\right) \\
&= M\left(A x_{2 n}, B x_{2 n+1}, q t\right) \\
& \geq \min M\left(T x_{2 n+1}, B x_{2 n+1}, t\right), M\left(S x_{2 n}, A x_{2 n}, t\right), M\left(S x_{2 n}, B x_{2 n+1},(1+\beta) t\right), \\
&\left.M\left(T x_{2 n+1}, A x_{2 n},(1-\beta) t\right), M\left(T x_{2 n+1}, S x_{2 n}, t\right)\right\} \\
&= \min \left\{M\left(y_{2 n+1}, y_{2 n+2}, t\right), M\left(y_{2 n}, y_{2 n+1}, t\right), M\left(y_{2 n}, y_{2 n+2},(1+\beta) t\right),\right. \\
&\left.M\left(y_{2 n+1}, y_{2 n+1},(1-\beta) t\right), M\left(y_{2 n+1}, y_{2 n}, t\right)\right\} \\
& \geq \min \{ M\left(y_{2 n+1}, y_{2 n+2}, t\right), M\left(y_{2 n}, y_{2 n+1}, t\right), \\
&\left.\Delta\left(M\left(y_{2 n}, y_{2 n+1}, t\right), M\left(y_{2 n+1}, y_{2 n+2}, \beta t\right)\right), 1, M\left(y_{2 n+1}, y_{2 n}, t\right)\right\} \\
&= \min \left\{M\left(y_{2 n+1}, y_{2 n+2}, t\right), M\left(y_{2 n}, y_{2 n+1}, t\right), 1, M\left(y_{2 n+1}, y_{2 n+2}, \beta t\right)\right\} .
\end{aligned}
$$

Taking $\beta \rightarrow 1$, we have

$$
M\left(y_{2 n+1}, y_{2 n+2}, q t\right) \geq \min \left\{M\left(y_{2 n+1}, y_{2 n+2}, t\right), M\left(y_{2 n}, y_{2 n+1}, t\right), 1\right\}
$$

By Remark 1.6,

$$
M\left(y_{2 n+1}, y_{2 n+2}, q t\right) \geq M\left(y_{2 n}, y_{2 n+1}, t\right)
$$

Similarly, we have

$$
M\left(y_{2 n+2}, y_{2 n+3}, q t\right) \geq M\left(y_{2 n+1}, y_{2 n+2}, t\right) .
$$

In general, for all $t>0$ and $n=1,2,3, \ldots$

$$
M\left(y_{n}, y_{n+1}, q t\right) \geq M\left(y_{n-1}, y_{n}, t\right)
$$

Thus for all $t>0$ and $n=1,2,3, \ldots$

$$
\begin{aligned}
M\left(y_{n}, y_{n+1}, q t\right) \geq & M\left(y_{n-1}, y_{n}, t\right) \\
\geq & M\left(y_{n-2}, y_{n-1}, t / q\right) \\
& \cdots \\
\geq & M\left(y_{1}, y_{2}, t / q^{n-2}\right) .
\end{aligned}
$$

Now, we show that $\left\{y_{n}\right\}$ is a Cauchy sequence in $X$.

Let $\sigma=\frac{q}{\mu}$. Since $0<\sigma<1$, the series $\sum_{i=1}^{\infty} \sigma^{i}$ is convergent and there exists $m_{0} \in \mathbb{N}$ such that $\sum_{i=m_{0}}^{\infty} \sigma^{i}<1$. Hence for every $m>m_{0}+1$ and $s \in \mathbb{N}$

$$
t>t \sum_{i=m_{0}}^{\infty} \sigma^{i}>t \sum_{i=m-1}^{m+s-1} \sigma^{i}
$$


Now

$$
\begin{aligned}
& M\left(y_{m+s+1}, y_{m}, t\right) \\
& \geq M\left(y_{m+s+1}, y_{m}, t \sum_{i=m-1}^{m+s-1} \sigma^{i}\right) \\
& \geq M\left(y_{m+s+1}, y_{m}, t \sigma^{m-1}+t \sigma^{m-1+1}+t \sigma^{m-1+2}+\cdots+t \sigma^{m-1+s}\right) \\
& \geq M\left(y_{m+s+1}, y_{m}, t \sigma^{m-1+1}+t \sigma^{m-1+2}+\cdots+t \sigma^{m-1+s}+t \sigma^{m-1}\right) \\
& \geq \Delta\left(M\left(y_{m+s+1}, y_{m+1}, t \sigma^{m-1+1}+t \sigma^{m-1+2}+\cdots+t \sigma^{m-1+s}\right)\right. \text {, } \\
& \left.M\left(y_{m+1}, y_{m}, t \sigma^{m-1}\right)\right) \\
& \geq \Delta\left(\Delta \left(M\left(y_{m+s+1}, y_{m+2}, t \sigma^{m-1+2}+\cdots+t \sigma^{m-1+s}\right),\right.\right. \\
& \left.\left.M\left(y_{m+2}, y_{m+1}, t \sigma^{m}\right), M\left(y_{m+1}, y_{m}, t \sigma^{m-1}\right)\right)\right) \\
& \geq \Delta\left(\Delta \left(\Delta \left(M\left(y_{m+s+1}, y_{m+3}, t \sigma^{m-1+3}+\cdots+t \sigma^{m-1+s}\right),\right.\right.\right. \\
& \left.\left.\left.M\left(y_{m+3}, y_{m+2}, t \sigma^{m+1}\right), M\left(y_{m+2}, y_{m+1}, t \sigma^{m}\right), M\left(y_{m+1}, y_{m}, t \sigma^{m-1}\right)\right)\right)\right) \\
& \geq \cdots \\
& \geq \overbrace{\Delta(\Delta(\cdots)}^{\text {s-times }}\left(M\left(y_{m+s+1}, y_{m+s}, t \sigma^{m-1+s}\right), M\left(y_{m+s}, y_{m+s-1}, t \sigma^{m+s-2}\right),\right. \\
& \left.\left.\left.\left.\cdots, M\left(y_{m+1}, y_{m}, t \sigma^{m-1}\right)\right)\right) \cdots\right)\right) \\
& \geq \overbrace{\Delta(\Delta(\cdots(\Delta)}^{\text {s-times }}\left(M\left(y_{1}, y_{2}, t \sigma^{m-1+s} / q^{m-1+s}\right), M\left(y_{1}, y_{2}, t \sigma^{m-2+s} / q^{m-2+s}\right)\right. \text {, } \\
& \left.\left.\left.\left.\cdots, M\left(y_{1}, y_{2}, t \sigma^{m-1} / q^{m-1}\right)\right)\right) \cdots\right)\right) \\
& \geq \overbrace{\Delta(\Delta(\cdots)}^{\text {s-times }}\left(M\left(y_{1}, y_{2}, t / \mu^{m-1+s}\right), M\left(y_{1}, y_{2}, t / \mu^{m-2+s}\right),\right. \\
& \left.\left.\left.\left.\cdots, M\left(y_{1}, y_{2}, t / \mu^{m-1}\right)\right)\right) \cdots\right)\right) \\
& \geq \Delta_{i=m-1}^{m+s-1} M\left(y_{1}, y_{2}, t / \mu^{i}\right) \\
& \geq \Delta_{i=m-1}^{\infty} M\left(y_{1}, y_{2}, t / \mu^{i}\right) \text {. }
\end{aligned}
$$

It is obvious that

$$
\lim _{n \rightarrow \infty} \Delta_{i=n}^{\infty} M\left(y_{1}, y_{2}, 1 / \mu^{i}\right)=1 \quad \text { implies } \quad \lim _{n \rightarrow \infty} \Delta_{i=n}^{\infty} M\left(y_{1}, y_{2}, t / \mu^{i}\right)=1
$$

for every $t>0$. Now for every $t>0$ and $\lambda \in(0,1)$, there exists $m_{1}(t, \lambda)$ such that $M\left(y_{m+s+1}, y_{m}, t\right)>1-\lambda$ for every $m \geq m_{1}(t, \lambda)$ and $s \in \mathbb{N}$. Hence $\left\{y_{n}\right\}$ is a Cauchy sequence in $X$. Since $X$ is complete, there exists a point $z$ in $X$ such that $\lim _{n \rightarrow \infty} y_{n}=z$ and this gives

$$
\lim _{n \rightarrow \infty} S x_{2 n}=\lim _{n \rightarrow \infty} T x_{2 n-1}=\lim _{n \rightarrow \infty} A x_{2 n-2}=\lim _{n \rightarrow \infty} B x_{2 n-1}=z
$$

for all $n \in \mathbb{N}$. 
Now, suppose that pair $(A, S)$ is compatible and $S$ is continuous. Then by Lemma 2.1, $A$ and $S$ are reciprocally continuous. Hence $\lim _{n \rightarrow \infty} S A x_{n}=S z$ and $\lim _{n \rightarrow \infty} A S x_{n}=A z$. Since pair (A, S) is compatible, we have

$$
\lim _{n \rightarrow \infty} M\left(A S x_{n}, S A x_{n}, t\right)=1
$$

and hence $M(A z, S z, t)=1$. Thus $A z=S z$.

Since $A(X) \subset T(X)$, there exists a point $v \in X$ such that $A z=S z=T v$. Take $\alpha=1$ in $(C 2)$, we have

$$
\begin{aligned}
& M(A z, B v, q t) \geq \min \{ M(T v, B v, t), M(S z, A z, t), M(S z, B v, t), \\
&M(T v, A z, t), M(T v, S z, t)\} \\
&=\min \{M(A z, B v, t), M(A z, A z, t), M(A z, B v, t), \\
& \\
&M(A z, A z, t), M(A z, A z, t)\} \\
& \geq M(A z, B v, t) .
\end{aligned}
$$

Thus by Lemma 1.11, we have $A z=B v$ and hence $A z=S z=T v=B v$. Since $A$ and $S$ are $R$-weakly commuting mappings, there exists $R>0$ such that $M(A S z, S A z, t) \geq(M A z, S z, t / R)=1$. Therefore, $A S z=S A z$ and $A A z=A S z=S A z=S S z$.

Similarly $B$ and $T$ are $R$-weakly commuting mappings, we have $B B v=$ $B T v=T B v=T T v$.

Again take $\alpha=1$, in $(C 2)$, we have

$$
\begin{aligned}
M(A A z, B v, q t) \geq \min \{ & M(T v, B v, t), M(S A z, A A z, t), M(S A z, B v, t), \\
& M(T v, A A z, t), M(T v, S A z, t)\} \\
= & \min \{M(T v, T v, t), M(A A z, A A z, t), M(A A z, A z, t), \\
\geq & M(A z, A A z, t), M(A z, A A z, t)\} \\
& M(A z, A z, t) .
\end{aligned}
$$

By Lemma 1.11, we have $A A z=A z=S A z$. Hence $A z$ is a common fixed point of $A$ and $S$. Similarly we can prove $B v=A z$ is a common fixed point of $B$ and $T$. Hence, $A z$ is a common fixed point of $A, B, S$ and $T$.

Finally, in order to prove the uniqueness, suppose that $A v(\neq A z)$ is another fixed point of $A, B, S$ and $T$. Then take $\alpha=1$ in $(C 2)$, we have

$$
\begin{aligned}
M(A z, A v, q t)= & M(A A z, B A v, q t) \\
\geq & \min \{M(T A v, B A v, t), M(S A z, A A z, t), M(S A z, B A v, t), \\
& M(T A v, A A z, t), M(T A v, S A z, t)\} \\
= & \min \{M(A v, A v, t), M(A z, A z, t), M(A z, A v, t), \\
& M(A v, A z, t), M(A v, A z, t)\} \\
\geq & M(A z, A v, t) .
\end{aligned}
$$


By Lemma 1.11, we have $A z=A v$. Therefore $A, B, S$ and $T$ have a unique common fixed point. This completes the proof.

If we put $S=T=I_{X}$ ( $I_{X}$ is the identity mapping) in Theorem 2.1, we get the following corollary.

Corollary 2.3. Let $(X, M, \Delta)$ be a fuzzy metric space with continuous minimum t-norm of Hadžić-type. Let $A$ and $B$ be reciprocally continuous selfmappings on $X$ satisfying

there exists $q \in(0,1)$ such that

$$
\begin{gathered}
M(A x, B y, q t) \geq \min \{M(y, B y, t), M(x, A x, t), M(x, B y,(2-\alpha) t), \\
M(y, A x, \alpha t), M(y, x, t)\}
\end{gathered}
$$

for every $x, y \in X$ and $t>0$, where $\alpha \in(0,2)$.

Then $A$ and $B$ have a unique common fixed point in $X$.

Example 2.4. Let $X=[2,20]$. Define for each $t>0$, define

$$
M(x, y, t)=\frac{t}{t+|x-y|}
$$

Then $(X, M, \Delta)$ is complete fuzzy metric space. Let $A, B, S$ and $T$ be selfmappings of $X$ defined as

$$
\begin{gathered}
A x=\left\{\begin{array}{ll}
2 & \text { if } x=2, \\
3 & \text { if } x>2,
\end{array} \quad B x= \begin{cases}2 & \text { if } x=2 \text { or } x>5, \\
6 & \text { if } 2<x \leq 5,\end{cases} \right. \\
S x=\left\{\begin{array}{ll}
2 & \text { if } x=2, \\
6 & \text { if } x>2,
\end{array} \quad T x= \begin{cases}2 & \text { if } x=2, \\
12 & \text { if } 2<x \leq 5, \\
x-3 & \text { if } x>5,\end{cases} \right.
\end{gathered}
$$

Then $A, B, S$ and $T$ satisfy all the conditions of Theorem 2.1 with $q \in(0,1)$ and have a unique common fixed point $x=2$.

Acknowledgements. One of the authors (Sanjay Kumar) would like to acknowledge University Grants Comission for providing financial grant Major Research Project under Ref. 39-41/2010(SR).

\section{References}

[1] M.A. Erceg, Metric space in fuzzy set theory, J. Math. Anal. Appl., 69 (1979), 205-230. http://dx.doi.org/10.1016/0022-247X(79)90189-6 
[2] A. George and P. Veeramani, On some results in fuzzy metric spaces, Fuzzy Sets and Systems, 64 (1994), 395-399. http://dx.doi.org/10.1016/01650114(94)90162-7

[3] M. Grabiec, Fixed points in fuzzy metric space, Fuzzy Sets Syestes, 27 (1988), 385-389. http://dx.doi.org/10.1016/0165-0114(88)90064-4

[4] O. Hadžić and E. Pap, Fixed Point Theory in Probabilistic Metric Spaces, Kluwer Academic Publishers, Dordrechi, 2001.

[5] G. Jungck, Compatible mappings and common fixed points, Internat J. Math. Math. Sci., 9 (1986), 771-779.

http://dx.doi.org/10.1155/S0161171286000935

[6] O. Kaleva and S. Seikkala, On fuzzy metric spaces, Fuzzy Sets and Systems, 12 (1984), 215-229.

http://dx.doi.org/10.1016/0165-0114(84)90069-1

[7] I. Kramosil and J. Michálek, Fuzzy metrics and statistical metric spaces, Kybernetika, 11 (1975), 336-344.

[8] S. N. Mishra, N. Sharma and S. L. Singh, Common fixed points of maps on fuzzy metric spaces, Internat. J. Math. Math. Sci., 17 (1994), 253-258. http://dx.doi.org/10.1155/S0161171294000372

[9] R. P. Pant, Common fixed point for noncommuting mapping, J. Math. Anal. Appl., 188 (1994), 436-440.

http://dx.doi.org/10.1006/jmaa.1994.1437

[10] R. P. Pant, A Common fixed point theorem under a new condition, Indian J. Pure Appl. Math., 30 (1999), 147-152.

[11] P.V. Subrahmanyam, Common fixed point theorems in fuzzy metric spaces, Inform. Sci., 83 (1995), 109-112. http://dx.doi.org/10.1016/00200255(94)00043-B

[12] R. Vasuki, Common fixed points for weakly commuting maps in fuzzy metric spaces, Indian J. Pure Appl. Math., 30 (1999), 419-423.

Received: June 17, 2014; Published: January 9, 2015 